\title{
Observations of Rabi oscillations in a non-polar InGaN quantum $\operatorname{dot}$
}

\author{
Benjamin P. L. Reid, ${ }^{1, *}$ Claudius Kocher, ${ }^{1,2}$ Tongtong Zhu, ${ }^{3}$ Fabrice Oehler, ${ }^{3}$ Robert \\ Emery ${ }^{3}$ Christopher C. S. Chan, ${ }^{1}$ Rachel A. Oliver ${ }^{3}$ and Robert A. Taylor ${ }^{1}$ \\ ${ }^{1}$ Department of Physics, University of Oxford, \\ Parks Road, Oxford, OX1 3PU, United Kingdom \\ ${ }^{2}$ Konstanz University, Germany \\ ${ }^{3}$ Department of Materials Science and Metallurgy, \\ 27 Charles Babbage Road Cambridge CB3 OFS, United Kingdom
}

\begin{abstract}
Experimental observation of Rabi rotations between an exciton excited state and the crystal ground state in a single non-polar InGaN quantum dot are presented. The exciton excited state energy is determined by photoluminescence excitation spectroscopy using two-photon excitation from a pulsed laser. The population of the exciton excited state is seen to undergo power dependent damped Rabi oscillations.
\end{abstract}

* benjamin.reid@physics.ox.ac.uk 


\section{INTRODUCTION}

Successful implementation of quantum information processing requires the ability to dynamically prepare, control and detect qubits. Coherent manipulation of a qubit can be achieved using a coherent optical field, the field can be used to control the amplitude and phase of the qubit superposition state $|\psi\rangle=\alpha|0\rangle+\beta|1\rangle$. For a driving optical field $E(t)$ acting on the qubit $|\psi\rangle$ in initial state $|0\rangle$, the post-pulse population of the state $|1\rangle$ will vary as $\sin ^{2}(\Theta / 2)$, where the pulse area is given by $\Theta(t)=(1 / \hbar) \int_{0}^{t} \mu E\left(t^{\prime}\right) d t^{\prime}$. This Rabi oscillation between states $|0\rangle$ and $|1\rangle$ is the signature of successful coherent manipulation of a qubit state.

Optical excitation has been used to demonstrate coherent control of both excitonic and spin qubit states in quantum dots in the III-As semiconductor system[1][2][3][4][5][6] and similar results have been obtained recently for the excitonic state in a GaN quantum dot emitting in the ultraviolet spectral range [7].

III-Nitride based quantum dots are promising candidates for qubits due to their ability to sustain excitonic emission at high temperatures, evidenced by single photon emission at $200 \mathrm{~K}$ from InGaN [8] and at $300 \mathrm{~K}$ from GaN quantum dots [9].

Demonstration of coherent control of quantum dot qubit states in the III-nitride system has been hampered by spectral diffusion caused by intrinsic electric field present in IIInitride quantum dots [10][11][12]. The non-centrosymmetric nature of the wurtzite crystal structure causes a polarisation discontinuity at the base of the quantum dot and thus an internal electric field which acts to decrease the oscillator strength of the exciton optical transition. In reporting successful coherent control of a GaN quantum dot, Holmes et. al. [7] use exceptionally small GaN quantum dots embedded at the tip of a GaN/AlGaN nanowire to reduce the influence of the internal electric field.

Another method shown to be a success in increasing the oscillator strength in InGaN quantum dots is growth on a non-polar plane [13]. Here the electric polarisation caused by the non-centrosymmetric crystal structure lies in the plane of the base of the quantum dot, and the angled side wall facets reduce the polarisation discontinuity at the side walls resulting in a reduced electric field and increased oscillator strength [14].

In this letter, we use non-polar $a$-plane (1120) InGaN quantum dots to show experimental evidence of power-dependent Rabi oscillations between an excitonic excited state $|1\rangle$ and 
the crystal ground state $|0\rangle$. After resonant excitation into the exciton excited state $|1\rangle$, the exciton relaxes into the excitonic ground state $|s\rangle$, and the photoluminescence (PL) from this state is used as an indirect measurement of the population of the excited state, enabling observation of Rabi oscillations in the blue spectral region which houses the fastest commercially available single photon detectors [15].

The quantum dot sample under study in this letter was grown by metal-organic vapor phase epitaxy in a Thomas-Swan $6 \times 2$ in. close-coupled showerhead reactor on $r$-plane (11̄02) $\mathrm{Al}_{2} \mathrm{O}_{3}$ substrates with trimethlygallium, trimethylinidum and ammonia as precursor gases. The a-plane GaN pseudo-substrates were prepared by epitaxial layer overgrowth as detailed elsewhere [16][17]. InGaN QDs were formed using a modified droplet epitaxy approach originally developed for $c$-plane InGaN quantum dots [18]. An InGaN epilayer was grown at $695^{\circ} \mathrm{C}$ and annealed at the same temperature in $\mathrm{N}_{2}$ atmosphere, before immediate capping with $\sim 10 \mathrm{~nm}$ GaN, followed by another $\sim 10 \mathrm{~nm}$ GaN growth at a temperature of $1050{ }^{\circ} \mathrm{C}$ in $\mathrm{H}_{2}$. A detailed growth methodology can be found elsewhere [13]. The quantum dot sample prepared in this way was then coated with $65 \mathrm{~nm} \mathrm{Au}$ and patterned with $1 \mu \mathrm{m}$ diameter circular apertures using electron beam lithography and a chemical wet etch. A pattern of such apertures was sufficient to enable optical isolation of single quantum dots.

The samples were mounted in a continuous helium flow cryostat (Janis ST-500) with a feedback loop temperature controller (Lakeshore 331) enabling cooling of the quantum dots to $4.2 \mathrm{~K}$. Individual QDs were addressed for microphotoluminescence using a confocal microscope with two-photon excitation from a mode-locked Ti:Sapphire laser (1 ps pulses at $76 \mathrm{MHz}$ ). The spectral resolution of the laser was measured to be $1.3 \mathrm{meV}$ and is sufficiently small to induced two-photon absorption above the InGaN band gap. We choose two-photon excitation as it has been shown to increase the ratio of individual quantum dot PL against the background PL signal from the underlying QW-like InGaN layer that is present as a consequence of the growth methodology [19][20]. The excitation laser pulses are focussed onto the sample using a $100 \times$ achromatic-corrected IR objective with NA 0.5. The objective is mounted on a piezo-controlled stage enabling nm positional precision. The emitted PL is then collected by the same objective and imaged onto a $25 \mu \mathrm{m}$ slit and dispersed by a 1200 lines/mm grating in a $0.3 \mathrm{~m}$ spectrometer (Shamrock 303i) and detected by a Peltier-cooled Si-based charge-coupled device (Andor). The setup as described gives a spectral resolution of $\sim 500 \mu \mathrm{eV}$ at typical InGaN QD emission energies. A diagram of the experimental setup 


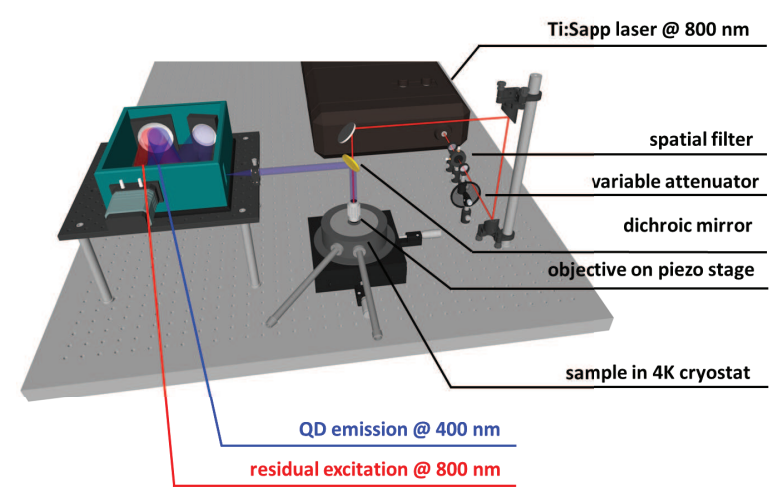

FIG. 1. Schematic of micro-photoluminescence setup. Single InGaN quantum dots can be excited via two photon absorption from a tunable Ti:Sapphire laser.

can be seen in figure 1 .

Photoluminescence excitation spectroscopy (PLE) was performed on individual quantum dots by tuning the energy of the Ti:Sapphire laser excitation and recording the response in intensity of the quantum dot under study. Micro-photoluminescence from an aperture is shown in figure 2, along with the photoluminescence excitation spectrum from the shaded quantum dot with PL emission at $2.83 \mathrm{eV}$. A broad resonance is seen around $3.1 \mathrm{eV}$ corresponding to absorption into continuum states formed by the underlying InGaN quantum well. Several resonances are seen at lower energies which could correspond to excited states of the quantum dot. QD excited states in close energetic proximity to the continuum states could give rise to a hybridization of the QD states and the continuum states [21] meaning the observation of Rabi oscillations is less likely. This could explain the fact that the PLE resonances closer to the continuum states are broader in energy. For this reason, the shaded narrow PLE resonance at $2.92 \mathrm{eV}$ is a suitable candidate to look for Rabi oscillations between this excited state $|1\rangle$ and the crystal ground state $|0\rangle$. The width of this resonance should correspond to the width of the PL peak at $2.83 \mathrm{eV}$ but it limited by the number of steps taken in varying the excitation energy.

The laser is tuned so that the energy of two photons from the laser is in resonance with the $|0\rangle \rightarrow|1\rangle$ transition and the PL from the quantum dot ground state $|s\rangle$ is used as an indirect measurement of the population of the excited state 1$\rangle$ as summarised in the inset of figure 2.

For two-photon excitation, the pulse area $\Theta$ is proportional to the excitation power $P$. 


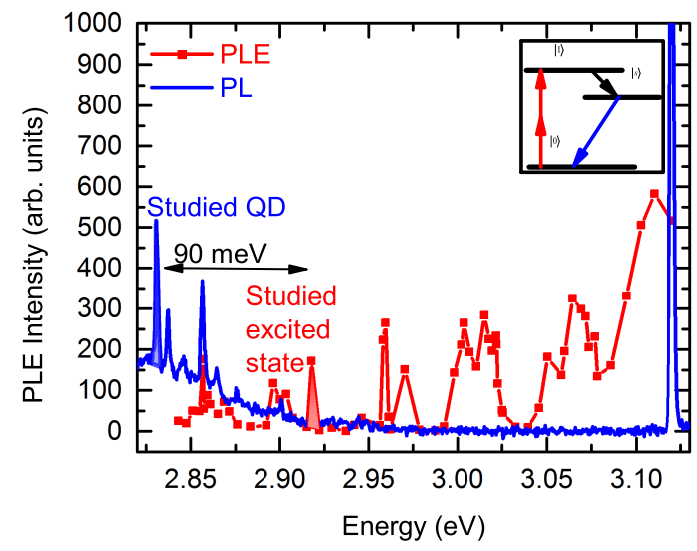

FIG. 2. PL spectrum from a single InGaN quantum dot (blue) overlaid with PLE spectrum (red) for the same quantum dot. The PLE spectrum shows several narrow resonances corresponding to excited states of the quantum dot and a broad resonance corresponding to the continuum states associated with the underlying quantum well layer. Inset: Energy level diagram showing the excitation and decay scheme used to observe Rabi oscillations.

Since GaN and InGaN are non-centrosymmetric, a second harmonic of the excitation laser is generated in the crystal and it's intensity (proportional to the square of the excitation power) can be can be used to measured the pulse area $\Theta$ in real time in some arbitrary units. The variation of the PL intensity of the QD ground state $|s\rangle$ with increasing pulse area is detailed in figure 3. In figure 3 (a) the micro-photoluminescence spectra from the studied quantum dot are presented with increasing pulse area for the case of resonant two-photon excitation $(|0\rangle \rightarrow|1\rangle)$. The QD intensity undergoes a clear oscillation with increasing pulse area while the intensity of the underlying quantum well layer follows the expected quadratic dependence on the pulse area. In contrast, figure 3 (b) shows the case of off-resonant excitation where both the $\mathrm{QD}$ and underlying quantum well increase with the square of the excitation power. After a certain excitation power the intensity saturates at a value corresponding to one emitted photon per laser pulse. The slight increase in PL intensity after saturation is consistent with repopulation of the quantum dot from the continuum quantum well states. Using a Lorentzian fit to each of the QD PL spectra to determine the intensity of the QD PL reveals damped Rabi oscillations as shown in figure 3 (c), where the pulse area is normalised so that the first peak in QD intensity corresponds to a value of $\pi$.

The power-dependent damping of the observed Rabi oscillations could have several 

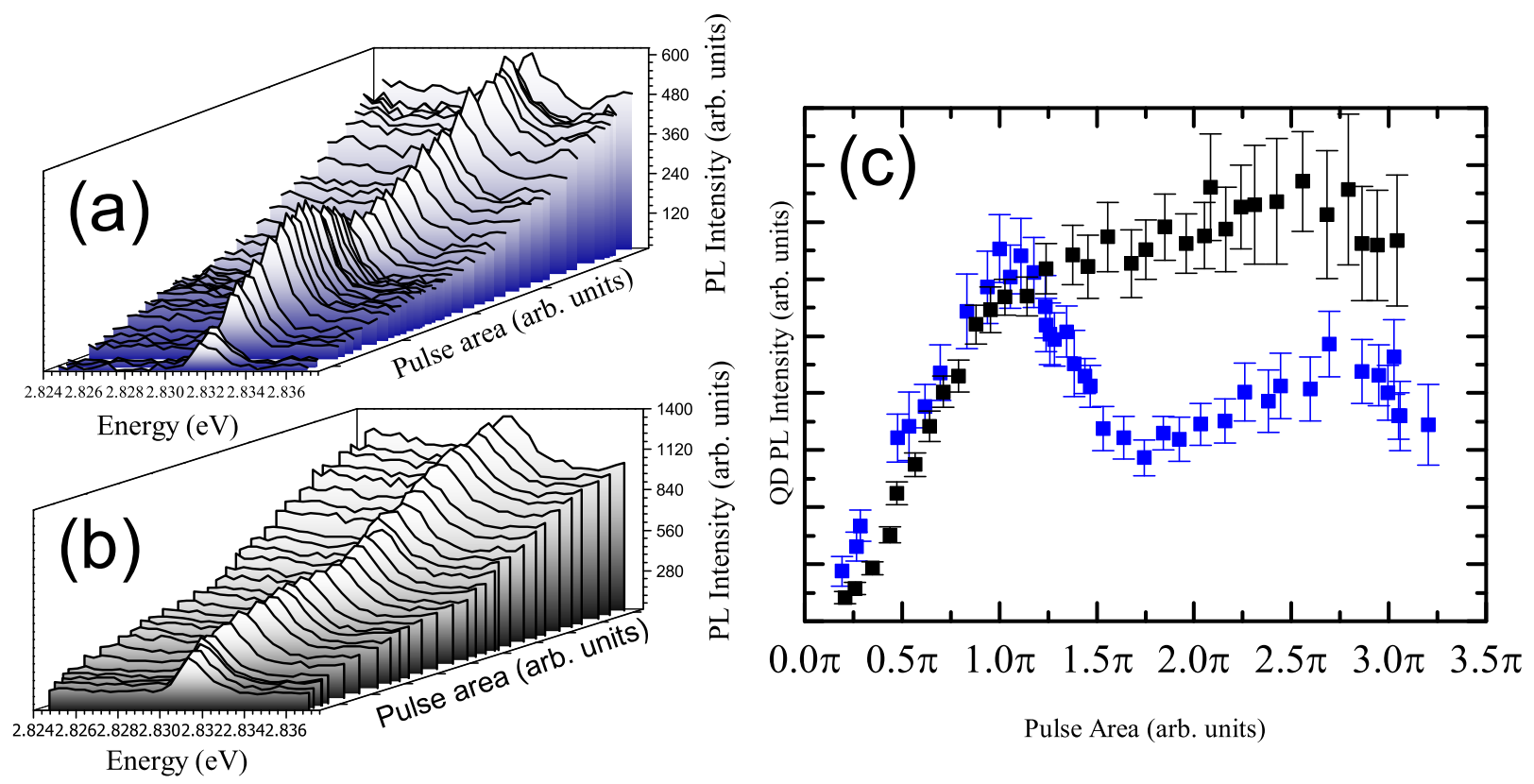

FIG. 3. (a) Resonant Case: Micro-photoluminescence spectra from a single InGaN quantum dot with increasing excitation power for resonant two-photon excitation. The intensity of the background quantum well luminescence increases as the square of the excitation power while the quantum dot PL undergoes shows an oscillation with increasing excitation power. (b) Non-resonant case: Non-resonant excitation of the same quantum dot produces the expected quadratic dependence of the quantum dot PL intensity on the excitation power. (c) QD PL intensity for the resonant excitation case (blue) and non resonant excitation case (black) as determined by Lorentzian fits to the PL spectra. The pulse area $\Theta(t)=(1 / \hbar) \int_{0}^{t} \mu E\left(t^{\prime}\right) d t^{\prime}$ is normalised such that $\pi$ corresponds to the first peak in the QD intensity for the case of resonant excitation. Damped Rabi oscillations are visible with increasing pulse area for resonant excitation, whereas a saturation of the PL intensity is measured for non-resonant excitation.

sources. Excitation in both the exciton excited state and the biexciton state would be a source of dephasing [22]. However, the energy difference of $90 \mathrm{meV}$ between exciton excited state and exciton ground state is much larger than typically measured for InGaN QDs $[23][24]$.

Despite the large energetic separation between the PLE resonance used as state $|1\rangle$ and the broad resonance corresponding to the continuum states of the quantum well, incoherent population of the quantum dot from these states could still be a source of dephasing [25][26], more-so as the excitation power is increased. 
Phonon interaction is another source of dephasing which may be pertinent in this case. Local heating of the sample at high excitation powers can result in both a power-dependent damping of the oscillation and a renormalisation of the oscillation period [27][28][29] as observed after $2 \pi$ in figure $3(\mathrm{c})$.

Another contribution to dephasing comes from the problem of spectral diffusion [10][12] - a spectral wandering of the exciton energy with time due to locally fluctuating charges changing the internal electric field in the quantum dot and thus affecting the energy levels via the quantum confined stark effect. The magnitude of spectral diffusion has been shown to increase with increasing excitation power [11] which would explain the power dependence of this effect.

Further investigations on the origins of the power-dependent damping measured here could reveal more closely the dominated mechanisms of dephasing in this system. At present, the dephasing time can be bounded by the laser pulse width (1 ps) and twice the lifetime of the excited state exciton.

In summary, we have measured power-dependent Rabi oscillations between the excited state exciton and the crystal ground state in a non-polar InGaN quantum dot. The increased oscillator strength of the exciton transition due to the non-polar nature of the InGaN QD is believed to play an important role in observing such Rabi oscillations. Such a system could be used in both quantum computation and single photon applications.

\section{ACKNOWLEDGMENTS}

This research was supported by the Engineering and Physical Sciences Research Council (EPSRC) U.K. (Grant No. EP/H047816/1).

[1] Y. Toda, T. Sugimoto, M. Nishioka, and Y. Arakawa, Applied Physics Letters 76, 3887 (2000).

[2] H. Htoon, D. Kulik, O. Baklenov, A. L. Holmes, T. Takagahara, and C. K. Shih, Phys. Rev. B 63, 241303 (2001).

[3] H. Kamada, H. Gotoh, J. Temmyo, T. Takagahara, and H. Ando, Phys. Rev. Lett. 87, 246401 (2001). 
[4] H. Htoon, T. Takagahara, D. Kulik, O. Baklenov, A. L. Holmes, and C. K. Shih, Phys. Rev. Lett. 88, 087401 (2002).

[5] L. Besombes, J. J. Baumberg, and J. Motohisa, Phys. Rev. Lett. 90, 257402 (2003).

[6] Q. Q. Wang, A. Muller, P. Bianucci, C. K. Shih, and Q. K. Xue, Applied Physics Letters 87, 031904 (2005).

[7] M. Holmes, S. Kako, K. Choi, P. Podemski, M. Arita, and Y. Arakawa, Phys. Rev. Lett. 111, $057401(2013)$.

[8] S. Deshpande, A. Das, and P. Bhattacharya, Applied Physics Letters 102, 161114 (2013).

[9] M. J. Holmes, K. Choi, S. Kako, M. Arita, and Y. Arakawa, Nano Letters 14, 982 (2014).

[10] J. W. Robinson, J. H. Rice, K. H. Lee, J. H. Na, R. A. Taylor, D. G. Hasko, R. A. Oliver, M. J. Kappers, C. J. Humphreys, and G. A. D. Briggs, Applied Physics Letters 86, 213103 (2005).

[11] R. Bardoux, T. Guillet, B. Gil, P. Lefebvre, T. Bretagnon, T. Taliercio, S. Rousset, and F. Semond, Phys. Rev. B 77, 235315 (2008).

[12] B. P. L. Reid, T. Zhu, T. J. Puchtler, L. J. Fletcher, C. C. S. Chan, R. A. Oliver, and R. A. Taylor, Japanese Journal of Applied Physics 52, 08JE01 (2013).

[13] T. Zhu, F. Oehler, B. P. L. Reid, R. M. Emery, R. A. Taylor, M. J. Kappers, and R. A. Oliver, Applied Physics Letters 102, 251905 (2013).

[14] S. Schulz, A. Berube, and E. P. O'Reilly, Phys. Rev. B 79, 081401 (2009).

[15] A. F. Jarjour, R. A. Oliver, and R. A. Taylor, Philosophical Magazine 87, 2077 (2007).

[16] M. Hberlen, T. J. Badcock, M. A. Moram, J. L. Hollander, M. J. Kappers, P. Dawson, C. J. Humphreys, and R. A. Oliver, Journal of Applied Physics 108, 033523 (2010).

[17] C. Johnston, M. Kappers, M. Moram, J. Hollander, and C. Humphreys, Journal of Crystal Growth 311, 3295 (2009).

[18] R. A. Oliver, G. A. D. Briggs, M. J. Kappers, C. J. Humphreys, S. Yasin, J. H. Rice, J. D. Smith, and R. A. Taylor, Applied Physics Letters 83, 755 (2003).

[19] A. F. Jarjour, T. J. Parker, R. A. Taylor, R. W. Martin, and I. M. Watson, physica status solidi (c) 2, 3843 (2005).

[20] A. F. Jarjour, A. M. Green, T. J. Parker, R. A. Taylor, R. A. Oliver, G. A. D. Briggs, M. J. Kappers, C. J. Humphreys, R. W. Martin, and I. M. Watson, Physica E: Low-dimensional Systems and Nanostructures 32, 119 (2006). 
[21] A. Vasanelli, R. Ferreira, and G. Bastard, Phys. Rev. Lett. 89, 216804 (2002).

[22] B. Patton, U. Woggon, and W. Langbein, Phys. Rev. Lett. 95, 266401 (2005).

[23] S. Amloy, Y. T. Chen, K. F. Karlsson, K. H. Chen, H. C. Hsu, C. L. Hsiao, L. C. Chen, and P. O. Holtz, Phys. Rev. B 83, 201307 (2011).

[24] J. W. Robinson, J. H. Rice, A. Jarjour, J. D. Smith, R. A. Taylor, R. A. Oliver, G. A. D. Briggs, M. J. Kappers, C. J. Humphreys, and Y. Arakawa, Applied Physics Letters 83, 2674 (2003).

[25] J. M. Villas-Bôas, S. E. Ulloa, and A. O. Govorov, Phys. Rev. Lett. 94, 057404 (2005).

[26] Q. Q. Wang, A. Muller, P. Bianucci, E. Rossi, Q. K. Xue, T. Takagahara, C. Piermarocchi, A. H. MacDonald, and C. K. Shih, Phys. Rev. B 72, 035306 (2005).

[27] J. Förstner, C. Weber, J. Danckwerts, and A. Knorr, Phys. Rev. Lett. 91, 127401 (2003).

[28] A. J. Ramsay, A. V. Gopal, E. M. Gauger, A. Nazir, B. W. Lovett, A. M. Fox, and M. S. Skolnick, Phys. Rev. Lett. 104, 017402 (2010).

[29] A. J. Ramsay, T. M. Godden, S. J. Boyle, E. M. Gauger, A. Nazir, B. W. Lovett, A. M. Fox, and M. S. Skolnick, Phys. Rev. Lett. 105, 177402 (2010). 